\title{
Theoretical and Experimental Study of the Air Flapper Valve Used for the Pulse Combustor
}

\author{
Zhiren Yin ${ }^{1,}$, Lijun Yang ${ }^{1, b}$ \\ ${ }^{1}$ School of Astronautics, Beihang University, Beijing, 100191, P.R. China \\ ayinzhr@gmail.com, byanglijun@buaa.edu.cn
}

Keywords: air flapper valve; mass flow rate; pulse combustor

\begin{abstract}
The basic process of air flapper valve was analyzed and the mass flow rate model of air flapper valve was established. The loss of flow through the air valve was considered. The opening process of the flapper was modeled with a non-viscous model, and the closing process was modeled with a viscous one. The model was validated the experimental data. The influence of the valve's space and the change of gas supply on mass flow rate were studied. The results show that the model can predict mass flow rate through air flapper valve effectively and there's a critical value of the valve's spacer, the drag coefficient $\lambda$ was constant under this value.
\end{abstract}

\section{Introduction}

The Helmholtz type pulse combustor is the most popular pulse combustion devices used in commercial and domestic areas. Air flapper valve is an important part to ensure the pulse combustion process.

The flapper valve controls the air entering the combustion chamber. And it prevents the hot back flow from the chamber. Because the pressure in the combustion chamber is periodic, it is difficult to calculate the mass flow rate of the flapper valve. The present literature about the flapper valve was not much, and most of them treated the valve as a simple orifice.

The model of the air flapper valve consisted two parts: mass flow rate model and flapper motion model. The mass flow rate model usually treated the valve as one dimensional orifice flow [1]. The losses during flowing were omitted, and a flow rate coefficient was used to refine the results. This method was used by Ahrens [2], Klicarslan [3], Temple [4] and Yu [5] et al.

The flapper motion model gave the information about the flapper position. Two kinds of the model were developed: non-viscous model and viscous model. Both of the two models were based on the Newton second law. Griffths and Weber used the non-viscous model and Temple used it only for the opening process of the valve [4]. The viscous model used is mostly linear viscous model which assumed that the friction was proportional to the velocity of the flapper. Dhar (1980), Lee, Van Essen, Morel and Zinn used the viscous model; Temple used this model for the closing process of the valve $[6,7]$.

In this paper, the working processes of the air flapper valve were analyzed. The influences of the main parameters were discussed.

\section{Model Setup}

The air flapper valve was consisted of the front plate, the back plate and the flapper, as depicted in Fig 1. From the time that the net pressure on the flapper was zero, the motion of the flapper can be described as four parts:

(1) As the pressure in the combustion chamber decreasing, the outer pressure was larger than the inner pressure. The flapper moved from $x=0$ to $x=x_{\max }$. The time during the opening process was $t_{\text {on }}$.

(2) The flapper stay at $x=x_{\max }$. The valve was fully open during the $t_{\text {full }}$.

(3) As the pressure in the combustion chamber increasing, the outer pressure was smaller than the inner pressure. The valve began to close form $x=x_{\max }$ to $x=0$. Back flow appeared before the valve was close. The time during the closing process was $t_{\text {off }}$.

(4) The flapper stay at $x=0$. The valve was fully open during the $t_{\mathrm{dr}}$. 


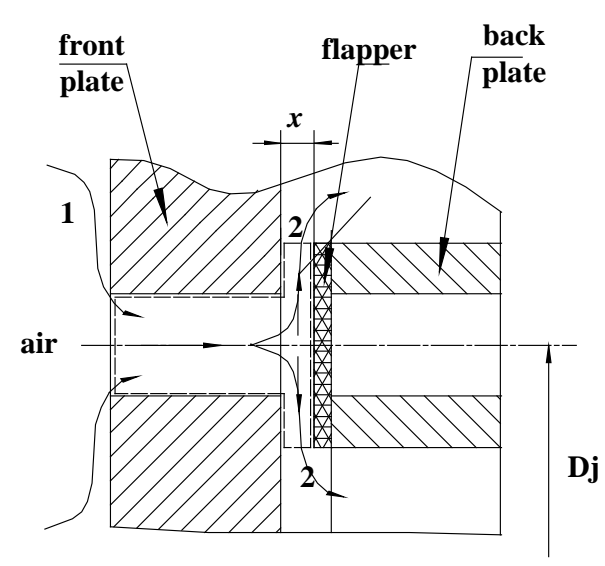

Fig. 1 Scheme of the air flapper valve.

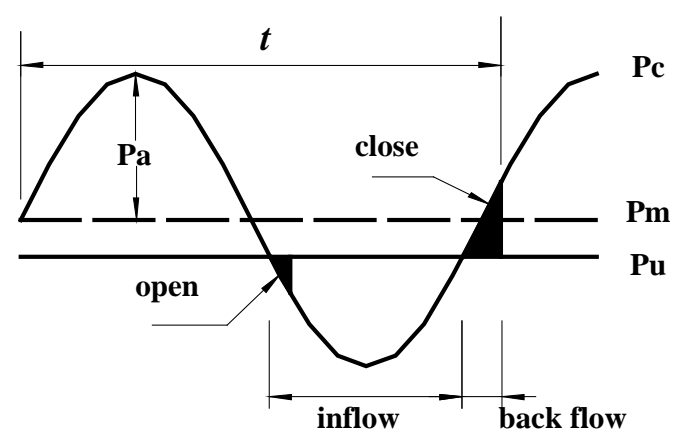

Fig. 2 Ideal valve characteristic.

Mass flow rate model. The mass flow rate during a cycle was decided by the first 3 processes:

$$
m_{\text {cycle }}=m_{\text {on }}+m_{\text {full }}-m_{\text {off }}=\int_{0}^{t_{\text {on }}} \dot{m}_{\text {on }}(t) d t+\int_{t_{\text {on }}}^{t_{\text {full }}} \dot{m}_{\text {full }}(t) d t+\int_{t_{\text {full }}}^{t_{\text {off }}} \dot{m}_{\text {off }}(t) d t
$$

The expression of the mass flow rate can be derived from the Bernoulli equation:

$$
\dot{m}=A_{2}\left(1-\frac{x}{x_{\max }}\right) \sqrt{\frac{2 \rho\left(p_{1}-p_{2}\right)}{1+\lambda}}
$$

Flapper motion model. The pressure in the combustion chamber can be simplified to a sinusoidal form:

$$
P_{\mathrm{c}}=P_{\mathrm{m}}+P_{\mathrm{a}} \sin (\omega t+\phi)
$$

Define $\tau=\omega t, \quad \chi=x / x_{\max }, \quad p_{n}=P / P_{\mathrm{a}}$ and

$$
p_{n, 0}=p_{\mathrm{n}, \mathrm{m}}-p_{\mathrm{n}, \mathrm{atm}}=\frac{P_{\mathrm{m}}}{P_{\mathrm{a}}}-\frac{P_{\mathrm{atm}}}{P_{\mathrm{a}}}, \quad \psi=\frac{P_{\mathrm{a}} A_{\mathrm{eff}}}{m \omega^{2} x_{\max }}
$$

The opening model was non-viscous. Applied the Newton second law to the flapper:

$$
m \frac{d^{2} x}{d t^{2}}=-P_{\text {net }} A_{\text {eff }}
$$

The boundary condition was

$$
t=0: \quad x=0 \text { and } d x / d t=0
$$

The motion of the flapper during the opening process can be solved:

$$
\chi=-\psi\left[\frac{1}{2} p_{n, 0} \tau^{2}+p_{n, 0}(\cos \tau-1)+(\sin \tau-\tau)\left(1-p_{n, 0}^{2}\right)^{0.5}\right]
$$

The closing model was viscous. Applied the Newton second law to the flapper: 


$$
m \frac{d^{2} x}{d t^{2}}=P_{\text {net }} A_{\text {eff }}-D \cdot \frac{d x}{d t}
$$

The boundary condition was

$$
t=0: \quad x=0 \text { and } d x / d t=0
$$

The motion of the flapper during the closing process can be solved:

$$
\begin{gathered}
\chi=e^{-\delta \tau}\left\{\left(\frac{\psi}{\delta}\right) e^{\delta \tau} \cos (\phi)+\left(\frac{p_{n, 0} \psi}{\delta^{2}}\right)(\delta \tau-1)-\psi e^{\delta \tau}\left(\frac{1}{\delta^{2}+1}\right)[\delta \sin (\tau+\phi)+\sin (\tau+\phi)]\right. \\
\left.+\left(\frac{p_{n, 0} \psi}{\delta^{2}}\right)+\left(\frac{\psi}{\delta}\right)\left(\frac{1}{\delta^{2}+1}\right)[\delta \sin (\tau)+\sin (\tau)]\right\}
\end{gathered}
$$

\section{Results and Discussion}

Fig. 3 shows the dependence of the mass flow rate $q$ on the valve gap $e$. When $\mathrm{e}<0.6$, the theoretical data agrees well with the experimental data. When $e=0.7$, the results were not good. The reason is that when e is large, the friction coefficient $\lambda$ become smaller. After modified to 0.33 at $\mathrm{e}=0.7$, the results agree again, as shown in Fig. 4.

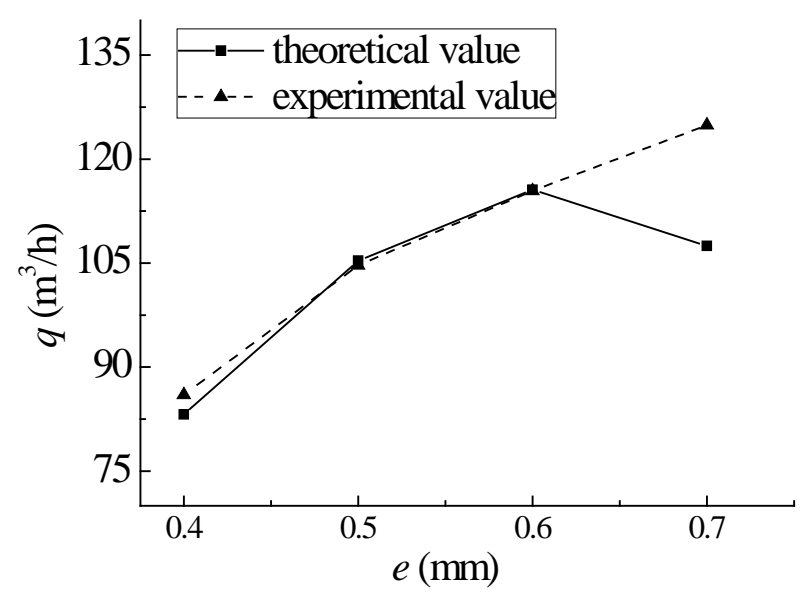

(a) $\lambda=0.9, \mathrm{De}=13$

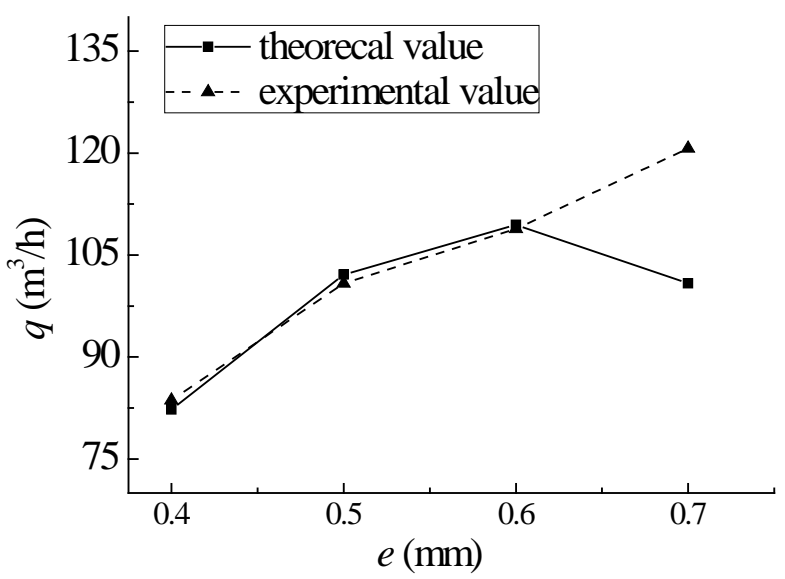

(b) $\lambda=0.9, \mathrm{De}=14$

Fig. 3 Dependence of the mass flow rate on the valve gap

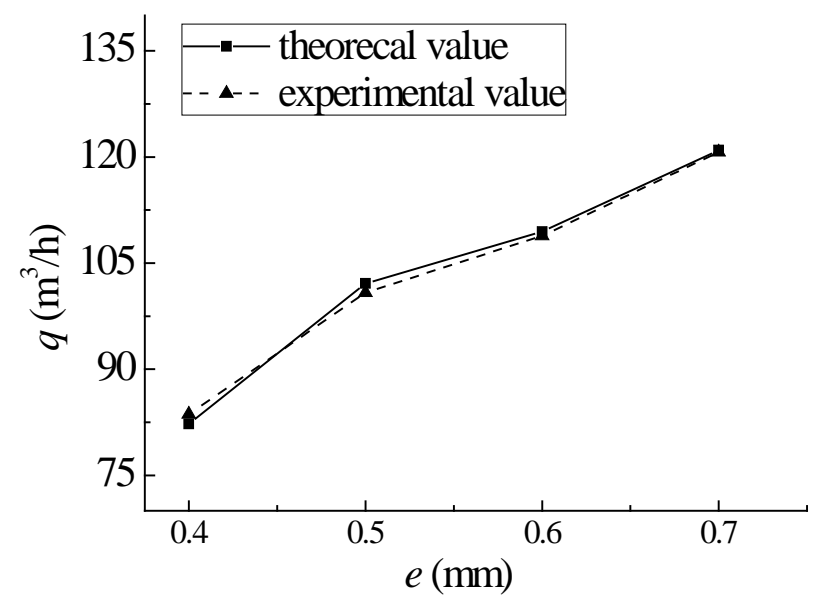

Fig. 4 Dependence of the modified mass flow rate on the valve gap 
There were two reasons for the increment of the air flow rate: the increment of the flow area and the change of the combustion chamber pressure. Fig. 5 shows the dependence of the pressure in combustion chamber on the valve gap. When $e$ was small, it did not influence the pressure much. As e increasing, the mean pressure and the pressure amplitude $\mathrm{Pa}$ increased significantly. $\mathrm{e}=0.6$ was the best work condition. When e>0.6, there was too much air supplement, Pa and Pm decrease.

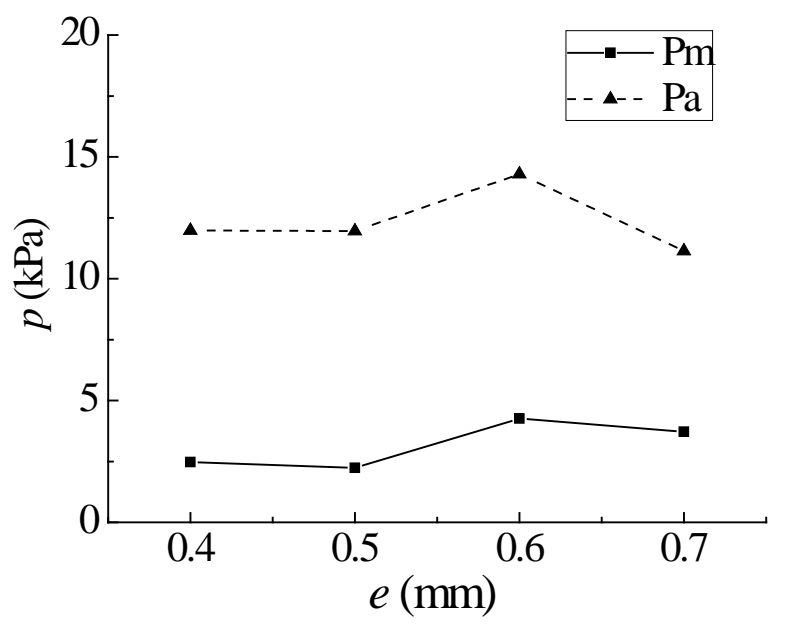

(a) $\mathrm{De}=13$

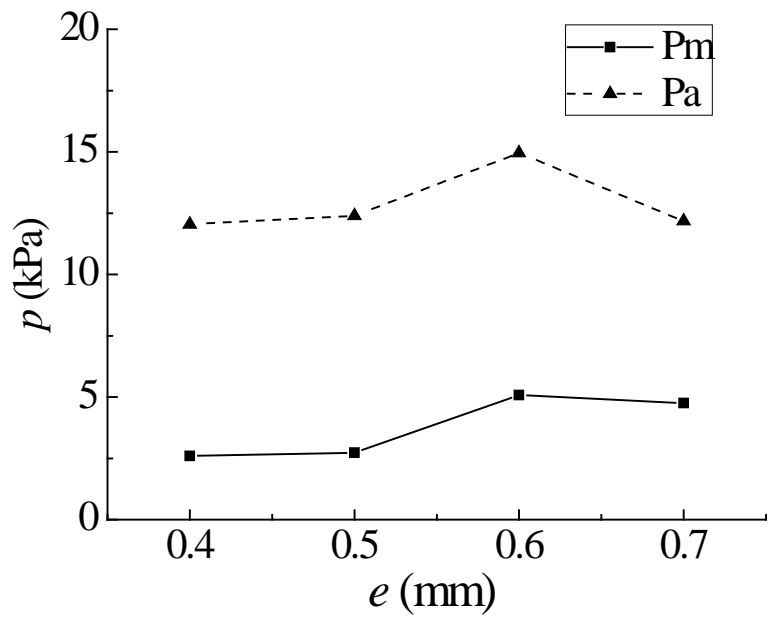

(b) $\mathrm{De}=14$

Fig. 5 Dependence of the pressure in combustion chamber on the valve gap

\section{Summary}

The model of the air flapper valve was set up and discussed. Results compared with the experimental data showed the effectiveness of the model. The mass flow rate of the air flapper valve was influenced both by the valve gap and the pressure in the combustion chamber. When using the valve model, it is necessary to choose a proper friction coefficient when the gap is large.

\section{References}

[1] ASHRAE Handbook, 2005 Fundamentals, Chapter 2, Fluid Flow. American Society of Heating, Refrigerating and Air-Conditioning Engineers, Inc., Atlanta, GA.

[2] F.W. Ahrens, C. Kim, and . Tam. SHRAE Transactions, Vol. 84, Part 1. (1978), p. 488-507.

[3] Ali Kilicarslan. International Journal of Energy Research, Vol. 29 (2005), p. 439-454.

[4] Temple K A. Thermal And Internal Acoustic Model Of A Helmholtz Type Pulse Combustion Furnace. Purdue University, 1996.

[5] G. Yu, M. Zhang and Z. Jiang, Gas and Heat, Vol. 4 (1994).

[6] Temple K, Goldschmidt V and Jones J. Evaluation of a Linearly Damped Model to Predict the Closing Motion of a Springless Flapper Valve. International Compressor Engineering Conference at Purdue, West Lafayette, Indiana. 1996.

[7] K.A. Temple, V. Goldschmidt and J. Jones. J. Vib. Acoust., Vol. 120(1998), p. 678-684. 